\title{
Treatment response in relation to subthreshold bipolarity in patients with major depressive disorder receiving antidepressant monotherapy: a post hoc data analysis (KOMDD study)
}

\author{
This article was published in the following Dove Press journal: \\ Neuropsychiatric Disease and Treatment \\ 17 May 2016 \\ Number of times this article has been viewed
}

\section{Young-Min Park' \\ Bun-Hee Lee ${ }^{2}$}

'Department of Psychiatry, Ilsan Paik Hospital, Inje University College of Medicine, Goyang, ${ }^{2}$ Department of Psychiatry, Seoul Eunpyeong Hospital, Seoul, Republic of Korea
Correspondence: Young-Min Park Department of Psychiatry, Ilsan Paik Hospital, Inje University College of Medicine, 2240, Daehwa-Dong, Ilsanseo-gu, Goyang 4I I-706,

Republic of Korea

$\mathrm{Tel}+82319107260$

Fax +82319107268

Email medipark@hanmail.net
Background: The aim of this observational study was to determine whether subthreshold bipolarity affects treatment response and remission in patients with major depressive disorder receiving antidepressant (AD) monotherapy over a 6-month follow-up period.

Methods: Seventy-eight patients with major depressive disorder were stratified into two subgroups according to the presence of subthreshold bipolarity, identified using the Korean version of the Mood Disorder Questionnaire (K-MDQ), which classifies patients as positive for a screening of bipolarity based on the cutoff for the total K-MDQ score (ie, 7 points). They received $\mathrm{AD}$ monotherapy such as escitalopram, sertraline, paroxetine, or tianeptine for 6 months. The Beck Depression Inventory (BDI), Hamilton Depression Rating Scale (HAMD), Hamilton Anxiety Scale, and Beck Scale for Suicide Ideation were applied at baseline, 1 week, 3 weeks, 2 months, 3 months, and 6 months.

Results: The mean HAMD, BDI, and Beck Scale for Suicide Ideation scores were higher in the bipolarity group than in the nonbipolarity group at 3 weeks. The mean BDI score was also higher in the bipolarity group than in the nonbipolarity group at 6 months. Evaluation of the ratio of improvement for each scale revealed different patterns of percentage changes between the two groups over the 6-month follow-up period. Furthermore, the response and remission rates (as assessed using BDI and HAMD scores) were higher in the nonbipolarity group than in the bipolarity group, with the exception of HAMD scores at the 3-week follow-up time point.

Conclusion: The findings of this study showed that depressed patients with bipolarity had a worse response to $\mathrm{AD}$ monotherapy than did those without bipolarity.

Keywords: subthreshold bipolarity, antidepressant, major depressive disorder, treatment response, remission

\section{Introduction}

Patients with major depressive episodes (MDEs) of bipolar disorder (BD) types I or II (BD I or BD II, respectively) generally exhibit weak responses to antidepressant (AD) therapy. ${ }^{1,2}$ In addition, most recent findings indicate that this high rate of resistance to ADs is not limited to BD I or II. Eighty percent of patients with AD-resistant unipolar depression were BD I, II, and not otherwise specified, and bipolar spectrum disorder (BSD) at 1-year follow-up. ${ }^{3}$ In an analysis of treatment-resistant depression, AD was associated with a poor outcome in both BD and BSD patients. ${ }^{4}$ Another study reported that patients with subthreshold bipolarity exhibited a poor prognosis over a 5-year follow-up period. ${ }^{5}$ Thus, the diagnosis of BD or BSD is underestimated, and the most 
common reason for treatment resistance appears to be masked BD. Furthermore, some investigators claimed that patients with Diagnostic and Statistical Manual of Mental Disorders, fourth edition (DSM-IV)-defined unipolar major depressive disorder (MDD) with subthreshold bipolar features should be excluded from drug trials. ${ }^{6}$

However, a recent meta-analysis found no significant difference in short-term AD response rates between patients with unipolar depression and BD. ${ }^{7}$ In addition, some investigators reported that fluoxetine monotherapy may be an effective short-term treatment for a BD II MDE with a relatively low rate of syndromal hypomanic episodes. ${ }^{8}$ These investigators also reported similar results in patients with rapid cycling BD II. ${ }^{9}$ In addition, some other investigators evaluated Sequenced Treatment Alternatives to Relieve Depression (STAR*D) study data, and reported that recent hypomanic- or manic-like symptoms, family history of BD, and early illness onset were not associated with treatment resistance to $\mathrm{AD}$, although irritability, psychic agitation, increased appetite, and psychotic-like symptoms were significantly associated with a worse treatment response to AD. ${ }^{10}$ Together these findings indicate that the effectiveness of ADs in patients with $\mathrm{BD}$ or $\mathrm{BSD}$ remains a matter of controversy.

The aim of this observational study was to determine whether subthreshold bipolarity affects treatment response and remission in patients with MDD - defined according to criteria in DSM-IV - receiving AD monotherapy over a 6-month follow-up period.

\section{Methods}

\section{Subjects and study design}

In total, 102 outpatients aged between 18 and 65 years who met the DSM-IV Text Revision criteria for MDD were recruited at Ilsan Paik Hospital for Korean MDD cohort for suicide prevention (KOMDD study). The following inclusion criteria of this cohort were applied: 1) having total Beck Depression Inventory (BDI) or Hamilton Depression Rating Scale (HAMD) scores exceeding 19 or 17, respectively, prior to the treatment and 2) no history of AD treatment within 12 weeks before first visit to our hospital. The exclusion criteria were the presence of 1) previous hypomanic/manic episode, 2) a high suicide risk, 3) history of brain trauma or organic brain disease, or 4) neurological disease. Depression severity was assessed using HAMD and BDI scores at baseline, 1 week, 3 weeks, 2 months, 3 months, and 6 months. Furthermore, the Hamilton Anxiety Scale, Beck Scale for Suicidal Ideation (BSS), and DSM-IV manic or hypomanic criteria for switch were applied at baseline, 1 week, 3 weeks,
2 months, 3 months, and 6 months. In addition, bipolarity screening was performed at baseline using the Korean version of the Mood Disorder Questionnaire (MDQ; K-MDQ), which classifies patients as positive for a screening of bipolarity based on a cutoff total K-MDQ score of 7 points.

Current data are a part of this KOMDD cohort. In addition, a post hoc analysis after a 6-month follow-up period was performed. Among initial 102 subjects, 78 subjects participated in MDD treatment course, which is similar to real-world setting for MDD pharmacotherapy (initially AD monotherapy). Based on clinical impression, subjects who need to receive augmentation or combination treatment afterward for analyzing the influence of bipolarity to $\mathrm{AD}$ monotherapy were excluded in the current analysis. After a 6-month follow-up period, 78 subjects were stratified into two groups according to the presence of subthreshold bipolarity, which was established using the K-MDQ, which classifies patients as positive for a screening of bipolarity based on a cutoff total K-MDQ score of 7 points, and history taking (nonbipolarity group $[\mathrm{n}=48]$ vs bipolarity group $[\mathrm{n}=30]$ ). The patients received AD monotherapy such as escitalopram, sertraline, paroxetine, or tianeptine for 6 months. Treatment response was defined as $\geq 50 \%$ improvement in mean HAMD or BDI scores from baseline. In addition, remission was defined as $\leq 7$ points of HAMD or $\leq 10$ points of BDI.

The study protocol was approved by the Ethics Committee of Ilsan Paik Hospital, and written informed consent to participate was obtained from all patients before beginning the investigation.

\section{Statistical analysis}

An observed case analysis was used to assess the efficacy during the 6-month follow-up period. Student's $t$-test and $\chi^{2}$ test were used to compare clinical variables between depressed patients with and without bipolarity. Repeatedmeasures analysis of variance was also conducted to analyze the differences in each scale between the two groups throughout the 6 months. In addition, Student's $t$-test was used to analyze the difference between the two groups in each scale at each visit.

\section{Results}

A total of 78 patients were enrolled; the completion rate of this study is $37.5 \%(18 / 48)$ in the nonbipolarity group and $26.6 \%(8 / 30)$ in the bipolarity group for 6 months. Table 1 compares the baseline clinical variables between the bipolarity and nonbipolarity groups. The mean baseline total BSS and K-MDQ scores differed significantly between the 
Table I Comparison of baseline clinical variables between groups with $(+)$ and without $(-)$ subthreshold bipolarity, defined according to a predefined cutoff (7 points) score on K-MDQ

\begin{tabular}{|c|c|c|c|}
\hline Variable & $\begin{array}{l}\text { Bipolarity }(-) \\
(n=48)\end{array}$ & $\begin{array}{l}\text { Bipolarity }(+) \\
(n=30)\end{array}$ & $P$-value \\
\hline Age (years) & $38.90 \pm 12.78$ & $39.13 \pm 15.31$ & 0.94 \\
\hline Sex (male/female) & $11 / 37$ & $6 / 24$ & 0.76 \\
\hline $\begin{array}{l}\text { Suicide attempt } \\
\text { history (no/yes) }\end{array}$ & $33 / 15$ & $13 / 17$ & $0.026 *$ \\
\hline Baseline BDI score & $25.75 \pm 9.52$ & $29.6 \pm 9.72$ & 0.089 \\
\hline Baseline HAMD score & $19.21 \pm 4.73$ & $18.5 \pm 5.39$ & 0.54 \\
\hline Baseline HAMA score & $20.3 I \pm 6.05$ & $22.07 \pm 6.31$ & 0.22 \\
\hline Baseline BSS score & $9.44 \pm 7.71$ & $16.67 \pm 9.97$ & $<0.0 I^{* *}$ \\
\hline K-MDQ score & $3.85 \pm 1.57$ & $9.63 \pm 2.01$ & $<0.0 I^{* *}$ \\
\hline
\end{tabular}

Notes: $* P<0.05, * * P<0.01$. Except where indicated otherwise, the data are mean \pm SD.

Abbreviations: BDI, Beck Depression Inventory; HAMD, Hamilton Depression Rating Scale; HAMA, Hamilton Anxiety Rating Scale; BSS, Beck Scale for Suicide Ideation; K-MDQ, Korean version of the Mood Disorder Questionnaire; SD, standard deviation.

bipolarity and nonbipolarity groups, as did the frequency of a history of suicide attempt. In contrast, the mean baseline total BDI, HAMD, and Hamilton Anxiety Scale scores did not differ significantly between the two groups. In addition, only one of the patients among the entire cohort experienced hypomanic conversion.

The mean HAMD, BDI, and BSS scores were higher in the bipolarity group than in the nonbipolarity group at 3 weeks (Figures 1-3). The mean BDI score was also higher in the bipolarity group than in the nonbipolarity group at 6 months (Figure 2). In particular, the mean BDI score in the

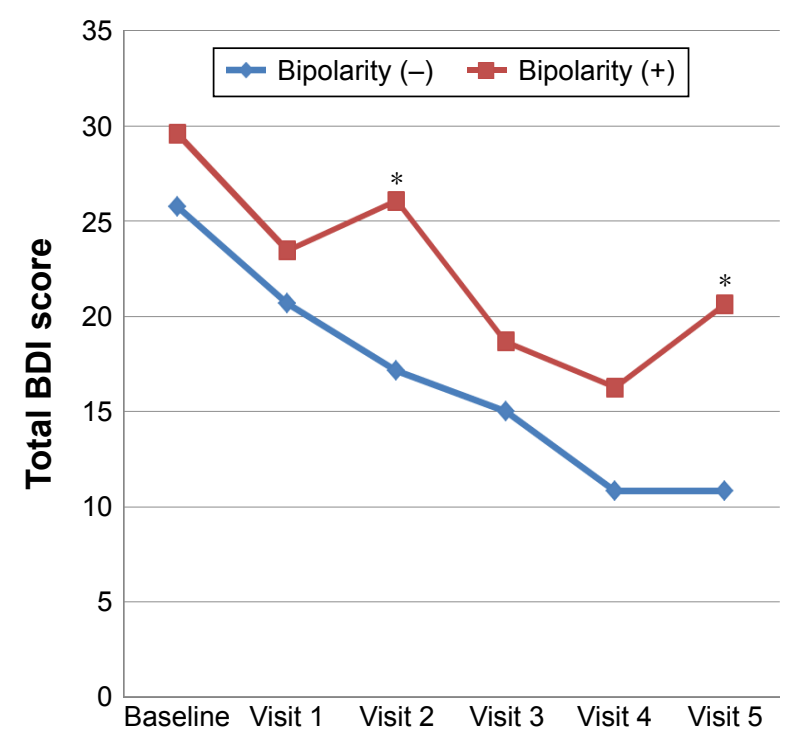

Figure I Comparison of mean BDI scores over a 6-month follow-up period between depressed patients with and without bipolarity.

Note: $* P<0.05$.

Abbreviation: BDI, Beck Depression Inventory.

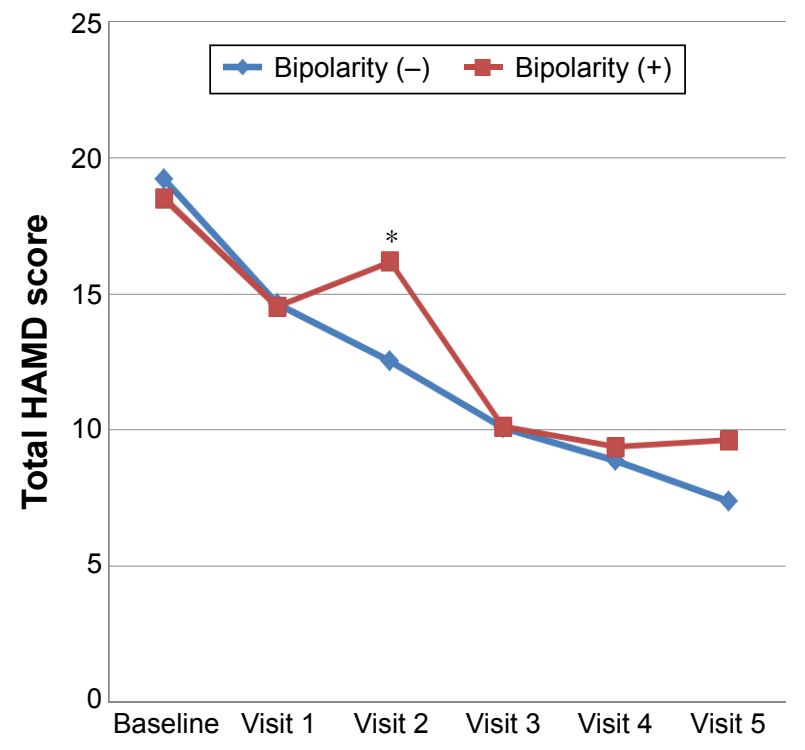

Figure 2 Comparison of mean HAMD scores over a 6-month follow-up period between depressed patients with and without bipolarity.

Note: $* p<0.05$

Abbreviation: HAMD, Hamilton Depression Rating Scale.

bipolarity group at the final visit (at 6 months) was more than 20 points. In addition, none of the scores on all scales differed significantly between the two groups at the 6-month follow-up on repeated analysis of variance. However, when the ratio of the change compared to scores attained at previous visits for each scale was evaluated (eg, visit $1 \mathrm{BDI}$ - visit $2 \mathrm{BDI} /$ visit 1 BDI $\times 100 \%$ ), different patterns emerged between the two groups over the 6-month follow-up period (Figures 4-7).

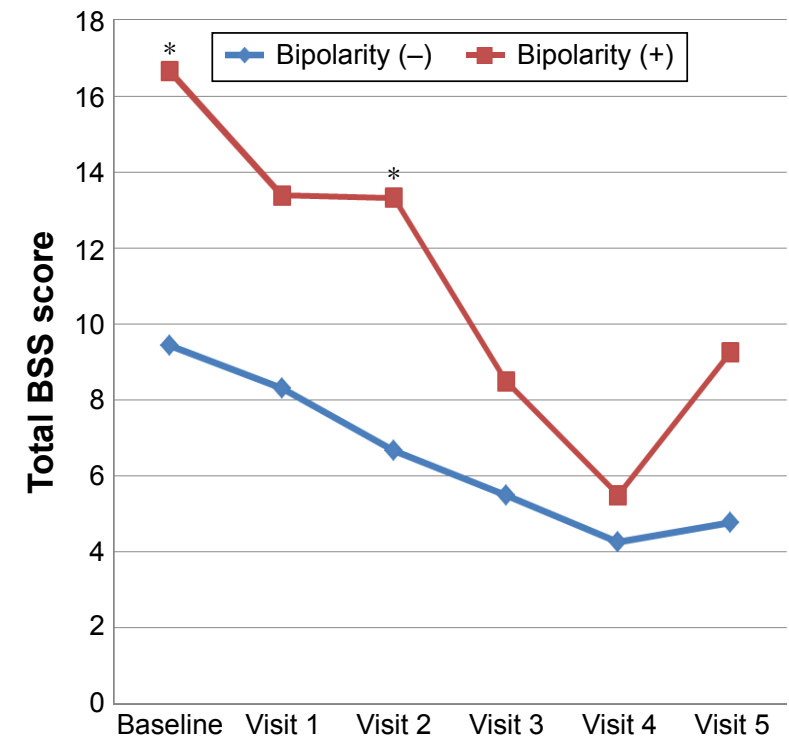

Figure 3 Comparison of mean BSS scores over a 6-month follow-up period between depressed patients with and without bipolarity.

Note: $* P<0.05$.

Abbreviation: BSS, Beck Scale for Suicide Ideation. 


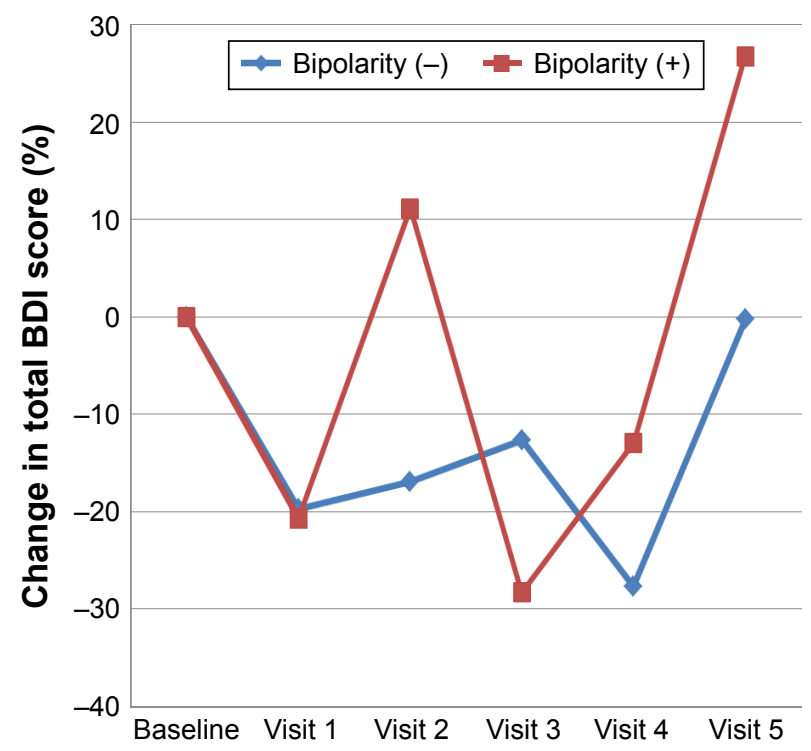

Figure 4 Comparison of the ratio of the change in mean BDI score relative to the score attained at the previous visit over the 6-month follow-up period between depressed patients with and without bipolarity.

Abbreviation: BDI, Beck Depression Inventory.

The response and remission rates of the nonbipolarity group, as assessed using BDI and HAMD scores, were higher than those of the bipolarity group, with the exception of those at the 3-week follow-up for HAMD (eg, the number needed to treat was typically three or four using either BDI or HAMD at 6 months; Table 2).

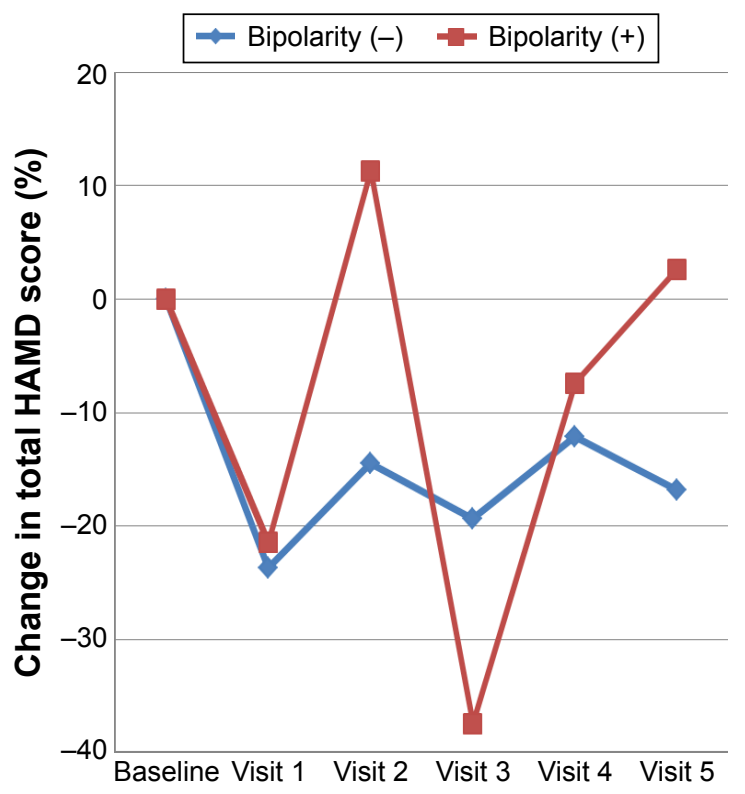

Figure 5 Comparison of the ratio of the change in mean HAMD score relative to the score attained at the previous visit over the 6-month follow-up period between depressed patients with and without bipolarity.

Abbreviation: HAMD, Hamilton Depression Rating Scale.

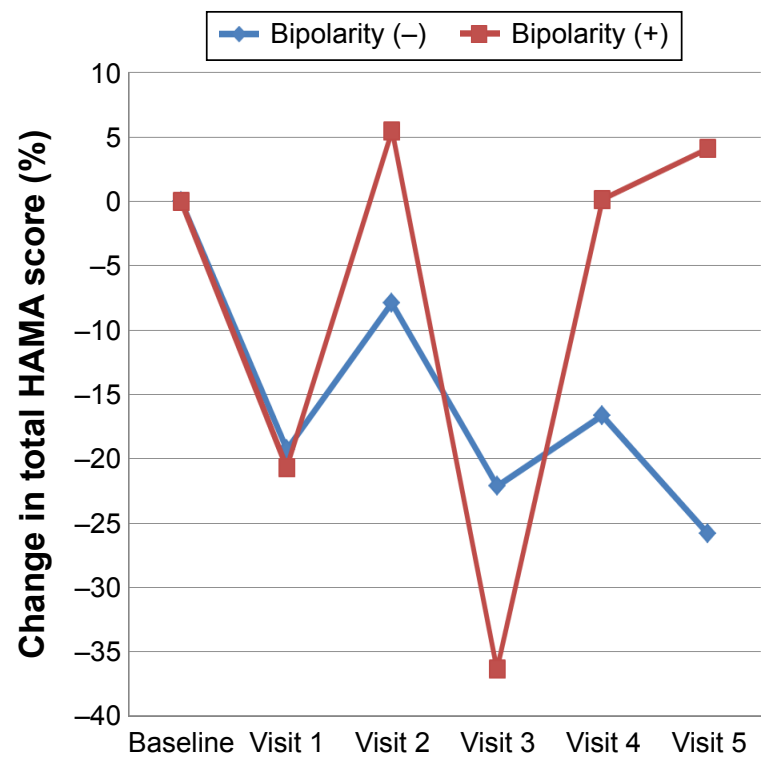

Figure 6 Comparison of the ratio of the change in mean HAMA score relative to the score attained at the previous visit over the 6-month follow-up period between depressed patients with and without bipolarity.

Abbreviation: HAMA, Hamilton Anxiety Rating Scale.

\section{Discussion}

This is the first study to evaluate whether subthreshold bipolarity affects treatment response and remission in patients with MDD receiving AD monotherapy over a 6-month follow-up period. The mean baseline total BSS and K-MDQ scores differed significantly between the bipolarity and nonbipolarity groups, as did the frequency of a history of

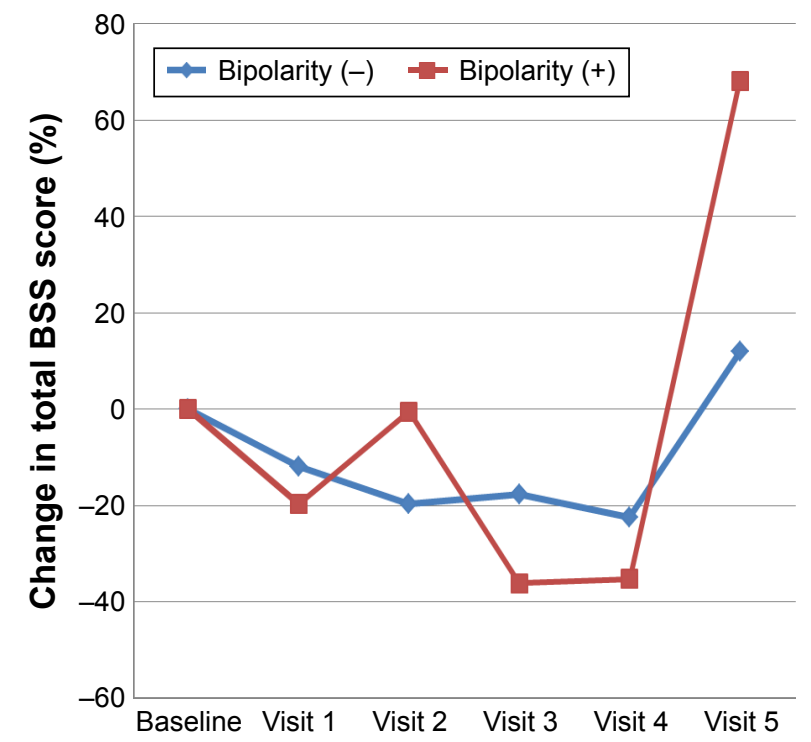

Figure 7 Comparison of the ratio of the change in mean BSS score relative to the score attained at the previous visit over the 6-month follow-up period between depressed patients with and without bipolarity.

Abbreviation: BSS, Beck Scale for Suicide Ideation. 
Table 2 Comparison of response and remission rates (as reflected by BDI and HAMD scores) between patients with and without bipolarity

\begin{tabular}{|c|c|c|c|c|}
\hline \multirow[t]{2}{*}{ Follow-up visit } & \multicolumn{2}{|l|}{ BDI } & \multicolumn{2}{|l|}{ HAMD } \\
\hline & Bipolarity (-) & Bipolarity (+) & Bipolarity (-) & Bipolarity (+) \\
\hline I week (partial response rate) & $45.8 \%$ & $36.4 \%$ & $45.0 \%$ & $32.0 \%$ \\
\hline 3 weeks (response rate) & $30.6 \%$ & $16.0 \%$ & $31.6 \%$ & $7.7 \%$ \\
\hline 2 months (remission rate) & $40.0 \%$ & $31.3 \%$ & $30.0 \%$ & $37.5 \%$ \\
\hline 3 months (remission rate) & $50.0 \%$ & $27.3 \%$ & $53.8 \%$ & $38.5 \%$ \\
\hline 6 months (remission rate) & $50.0 \%$ & $25.0 \%$ & $66.7 \%$ & $37.5 \%$ \\
\hline
\end{tabular}

Abbreviations: BDI, Beck Depression Inventory; HAMD, Hamilton Depression Rating Scale.

suicide attempt. Thus, these patients with bipolarity seem to be vulnerable to suicide.

MDD patients with subthreshold bipolarity had a higher total BDI score than those without subthreshold bipolarity at both 3 weeks and 6 months. In particular, the mean BDI score of the bipolarity group was more than 20 points at 6 months, which indicates relapse of depression with moderate severity. In addition, the response and remission rates in the nonbipolarity group, as assessed using BDI and HAMD scores, were higher than in the bipolarity group, with the exception of the 3-week follow-up score for HAMD. Thus, the bipolarity group appeared to respond worse to AD monotherapy than the nonbipolarity group. These results corresponded with previous studies, ${ }^{11-13}$ which found that underlying bipolarity was an important contributor to $\mathrm{AD}$ nonresponse. Furthermore, Correa et al reviewed 196 articles and found a high rate of conversion of treatment-resistant depression into BD. ${ }^{4}$ Maalouf et al also found that depressed patients with subsyndromal manic symptoms responded less to $\mathrm{AD}$ than those without the same symptoms, although that study only involved adolescents. ${ }^{14}$

In addition to manic-like symptoms, O'Donovan et al found that patients with bipolarity including family history of BD or suicide, early onset, mixed symptoms, and agitation exhibited a lower rate of response to AD monotherapy compared to patients without bipolarity, although that study was retrospective. ${ }^{15}$ Recently, BD I differed from MDD in terms of greater rates of psychomotor retardation and psychotic features. ${ }^{16}$ In addition, BD II was distinguished from MDD by the greater likelihood of mixed features. ${ }^{16}$ However, a previous $(\mathrm{STAR} * \mathrm{D})$ post hoc analysis revealed that while psychosis and depression severity were associated with treatment resistance, a family history of BD and manic-like symptoms were not. ${ }^{10}$ In addition, the BSD criteria suggested by Ghaemi et $\mathrm{al}^{17}$ did not predict treatment resistance, and some investigators found that fluoxetine monotherapy may be a more effective and tolerable treatment in patients with
BD II than lithium. ${ }^{8}$ Thus, the relationship between treatment resistance and $\mathrm{BD}$ or bipolarity remains unclear.

Intriguingly, the changes in the four scales in the bipolarity group exhibited a wax-and-wane pattern with a "W" shape, which was not seen in the nonbipolarity group (Figures 3-7). This reflects an alternation of improvement and aggravation in the bipolarity group when they were evaluated over a shortterm period; for example, there was a 10\% aggravation of HAMD score from visit 2 to visit 3, but an approximate $40 \%$ improvement on the same scale from visit 3 to visit 4 in that patient group (Figure 5). Thus, clinicians must identify the presence of subthreshold bipolarity in patients with MDD and will then know to expect this waxing and waning pattern in their treatment response to AD monotherapy and a low remission rate in that patient group.

The findings regarding manic or hypomanic switch with $\mathrm{AD}$ use in $\mathrm{BD}$ are also controversial. Some investigators have found that AD can cause (hypo)manic switch in BD during acute depressive and maintenance phase. ${ }^{18,19}$ However, hypomanic switch occurred in only one patient with subthreshold bipolarity in the present study. Bond et al found that the risk of (hypo)manic switch with AD use in BD II is intermediate between that in BD I and MDD. ${ }^{20}$ Thus, it is possible that the risk of (hypo)manic switch depends on the subtype of BD. However, in the large, randomized, controlled Systematic Treatment Enhancement Program for Bipolar Disorder (STEP-BD) study, there was no difference in the rate of (hypo)manic switch or in the efficacy between mood stabilizer plus AD (eg, sertraline or bupropion) and mood stabilizer plus placebo in patients with BDs I and II. ${ }^{2}$ However, the patients in both the groups of that study were allowed to use other drugs with an AD effect, such as quetiapine, olanzapine, and lamotrigine, as well as mood stabilizers. ${ }^{21}$ Many clinicians still prefer to prescribe a combination treatment of mood stablilizer and $\mathrm{AD}$ to depressed patients with BD. In addition, in one meta-analysis, the pooled relative risk of (hypo)manic switch indicated a lack 
of significant difference in responses of unipolar and $\mathrm{BD}$ patients in acute MDEs. ${ }^{7}$ Thus, the available evidence is not sufficient to draw the conclusion that ADs such as selective serotonin-reuptake inhibitors should not be used in patients with BD or bipolarity due to manic or hypomanic switch. However, the findings of the present study suggest that the reason that care should be taken when prescribing ADs (and especially selective serotonin-reuptake inhibitors) in patients with bipolarity is not AD tolerability (eg, manic switch), but rather efficacy.

This study was subject to several limitations. K-MDQ played an important role in dividing the patients into two groups. However, scores on that scale are not specific to BD; Zimmerman et al found that while MDQ mainly identifies mood instability, as opposed to distinct episodes of abnormal mood, many patients who score positive on the screen meet the criteria for borderline personality disorder. ${ }^{22}$ However, even if it is accepted that MDQ positivity does not necessarily reflect the presence of $\mathrm{BD}$, in this (MDQ-positive) subgroup, the finding that the AD response was lower has clinical significance. In addition, patients with borderline personality disorder were excluded in our study. Second, the sample was small and the study did not have a randomized design. Third, the number of comparisons probably can affect type I error. Notwithstanding these limitations, the present study was performed in a real clinical setting. Randomized and controlled studies with large samples are needed to confirm the present findings.

\section{Acknowledgments}

This study was supported by a grant from the National Research Foundation of Korea (NRF), funded by the Ministry of Education (NRF-2014R1A1A2A10059345). The authors would like to thank Jo BW for her assistance with data collection.

\section{Disclosure}

The authors report no conflicts of interest in this work.

\section{References}

1. Pacchiarotti I, Bond DJ, Baldessarini RJ, et al. The International Society for Bipolar Disorders (ISBD) task force report on antidepressant use in bipolar disorders. Am J Psychiatry. 2013;170(11):1249-1262.

2. Sachs GS, Nierenberg AA, Calabrese JR, et al. Effectiveness of adjunctive antidepressant treatment for bipolar depression. $N$ Engl J Med. 2007;356(17):1711-1722.

3. Sharma V, Khan M, Smith A. A closer look at treatment resistant depression: is it due to a bipolar diathesis? J Affect Disord. 2005;84(2-3): 251-257.
4. Correa R, Akiskal H, Gilmer W, Nierenberg AA, Trivedi M, Zisook $\mathrm{S}$. Is unrecognized bipolar disorder a frequent contributor to apparent treatment resistant depression? J Affect Disord. 2010;127(1-3): $10-18$.

5. Maj M, Pirozzi R, Magliano L, Fiorillo A, Bartoli L. Agitated "unipolar" major depression: prevalence, phenomenology, and outcome. J Clin Psychiatry. 2006;67(5):712-719.

6. Rihmer Z, Dome P, Gonda X. Antidepressant response and subthreshold bipolarity in "unipolar" major depressive disorder: implications for practice and drug research. J Clin Psychopharmacol. 2013;33(4): 449-452.

7. Vazquez GH, Tondo L, Undurraga J, Baldessarini RJ. Overview of antidepressant treatment of bipolar depression. Int J Neuropsychopharmacol. 2013;16(7):1673-1685.

8. Amsterdam JD, Shults J. Efficacy and mood conversion rate of shortterm fluoxetine monotherapy of bipolar II major depressive episode. J Clin Psychopharmacol. 2010;30(3):306-311.

9. Amsterdam JD, Luo L, Shults J. Effectiveness and mood conversion rate of short-term fluoxetine monotherapy in patients with rapid cycling bipolar II depression versus patients with nonrapid cycling bipolar II depression. J Clin Psychopharmacol. 2013;33(3):420-424.

10. Perlis RH, Uher R, Ostacher M, et al. Association between bipolar spectrum features and treatment outcomes in outpatients with major depressive disorder. Arch Gen Psychiatry. 2011;68(4):351-360.

11. Woo YS, Chae JH, Jun TY, Kim KS, Bahk WM. The bipolar diathesis of treatment-resistant major depressive disorder. Int J Psychiatry Clin Pract. 2008;12(2):142-146.

12. Li CT, Bai YM, Huang YL, et al. Association between antidepressant resistance in unipolar depression and subsequent bipolar disorder: cohort study. Br J Psychiatry. 2012;200(1):45-51.

13. Dudek D, Siwek M, Zielinska D, Jaeschke R, Rybakowski J. Diagnostic conversions from major depressive disorder into bipolar disorder in an outpatient setting: results of a retrospective chart review. $J$ Affect Disord. 2013;144(1-2):112-115.

14. Maalouf FT, Porta G, Vitiello B, et al. Do sub-syndromal manic symptoms influence outcome in treatment resistant depression in adolescents? A latent class analysis from the TORDIA study. $J$ Affect Disord. 2012;138(1-2):86-95.

15. O'Donovan C, Garnham JS, Hajek T, Alda M. Antidepressant monotherapy in pre-bipolar depression; predictive value and inherent risk. J Affect Disord. 2008;107(1-3):293-298.

16. Frankland A, Cerrillo E, Hadzi-Pavlovic D, et al. Comparing the phenomenology of depressive episodes in bipolar I and II disorder and major depressive disorder within bipolar disorder pedigrees. J Clin Psychiatry. 2015;76(1):32-38; quiz 39.

17. Ghaemi SN, Ko JY, Goodwin FK. "Cade's disease" and beyond: misdiagnosis, antidepressant use, and a proposed definition for bipolar spectrum disorder. Can J Psychiatry. 2002;47(2):125-134.

18. Sidor MM, MacQueen GM. An update on antidepressant use in bipolar depression. Curr Psychiatry Rep. 2012;14(6):696-704.

19. Pacchiarotti I, Valenti M, Colom F, et al. Differential outcome of bipolar patients receiving antidepressant monotherapy versus combination with an antimanic drug. J Affect Disord. 2011;129(1-3):321-326.

20. Bond DJ, Noronha MM, Kauer-Sant'Anna M, Lam RW, Yatham LN. Antidepressant-associated mood elevations in bipolar II disorder compared with bipolar I disorder and major depressive disorder: a systematic review and meta-analysis. J Clin Psychiatry. 2008;69(10): 1589-1601.

21. Nolen WA. The continuum of unipolar depression - bipolar II depression - bipolar I depression: different treatments indicated? World Psychiatry. 2011;10(3):196-197.

22. Zimmerman M. Would broadening the diagnostic criteria for bipolar disorder do more harm than good? Implications from longitudinal studies of subthreshold conditions. J Clin Psychiatry. 2012;73(4): 437-443. 
Neuropsychiatric Disease and Treatment

Dovepress

\section{Publish your work in this journal}

Neuropsychiatric Disease and Treatment is an international, peerreviewed journal of clinical therapeutics and pharmacology focusing on concise rapid reporting of clinical or pre-clinical studies on a range of neuropsychiatric and neurological disorders. This journal is indexed on PubMed Central, the 'PsycINFO' database and CAS,

and is the official journal of The International Neuropsychiatric Association (INA). The manuscript management system is completely online and includes a very quick and fair peer-review system, which is all easy to use. Visit http://www.dovepress.com/testimonials.php to read real quotes from published authors.

Submit your manuscript here: http://www.dovepress.com/neuropsychiatric-disease-and-treatment-journal 